\title{
Recent Advances in Experimental and Numerical Analysis of Combustor Flow Fields in Supersonic Flow Regime
}

\author{
K.M.Pandey, Member IACSIT and A.P. Singh
}

\begin{abstract}
Survey of experimental and numerical studies have been done for different complex flow fields with respect to different aspect by mixing of different types of fuel and mixture with high speed flows in the combustion chamber. In this field many researchers worked to develop a configuration giving efficient mixing and combustion, also meeting the requirements of flame holding and completion of combustion with sufficient stabilization in the flow field. The main attention is paid to the local intensity of heat release, which determines, together with the duct geometry, techniques for flame initiation and stabilization, injection techniques and quality of mixing the fuel with oxidizer, the gas-dynamic flow regime. From the survey it concluded that some area in which more attention to be paid like total pressure loss in combustion chamber and design a contour to make the flow at Mach number 2 for various higher Mach numbers for supersonic combustion and analyze the flow properties in combustion chamber and its effect by using the variety of fuel with the help of CFD tool.
\end{abstract}

Index Terms-Complex flow, contour design, , flame holding, high speed flow, total pressure loss

\section{INTRODUCTION}

Supersonic combustion is the key enabling technology for sustained hypersonic flights. In scramjet engines of current interest, the combustor length is typically of the order of $1 \mathrm{~m}$, and the residence time of the mixture is of the order of milliseconds. Due to the high supersonic flow speed in the combustion chamber, problems arise in the mixing of the reactants, flame anchoring and stability and completion of combustion within the limited combustor length. The flow field in the scramjet combustor is highly complex. It is shown that when the flight speed is low, the kinetic energy of the air is not enough to be used for the optimal compression. Further compression by machines is needed in order to obtain a higher efficiency. For example, a turbojet employs a turbine machine for further compression. When the flight speed is higher than a certain value, the air flow entering a combustor will remain to be supersonic after the optimal compression. With a further compression (i. e. deceleration), the efficiency of the engine will decrease. Therefore the combustion has to take place under the supersonic flow condition. This kind of air-breathing engine, which works under hypersonic flight condition, is called the supersonic combustion ramjet (Scramjet). The term of "supersonic combustion" applied here means the combustion in a supersonic flow. The efficiency of heat supply to the combustion chamber based on the analysis of literature data on combustion processes in a confined high-velocity and high-temperature flow for known initial parameters is considered.This was given by Mr.P.K.Tretyakov[1]. The process efficiency is characterized by the combustion completeness and total pressure losses. The main attention is paid to the local intensity of heat release, which determines, together with the duct geometry, techniques for flame initiation and stabilization, injection techniques and quality of mixing the fuel with oxidizer, the gas-dynamic flow regime. The study of supersonic combustion of hydrogen has been conducted by Shigeru Aso\& Arifnur Hakim et al. [2] using a reflected-type shock tunnel which generated a stable supersonic air flow of Mach number of 2 with the total temperature of $2800 \mathrm{~K}$ and the total pressure of $0.35 \mathrm{MPa}$. He concluded that The Schlieren images show that the increase of injection pressure generated strong bow shock, resulting in the pressure loses.

Supersonic combustion data obtained at the low static temperatures appropriate for an efficient scramjet engine are reviewed by T.Cain and C. Walton[3]. Attention is focused at the methods by which the fuel was ignited and combustion maintained. This is particularly common for supersonic combustion experiments and many examples are found in the literature of experiments conducted with inlet temperatures much higher than practical in flight. There is a good reason for this: it is difficult to sustain a hydrogen or hydrocarbon flame in a low temperature supersonic flow. A well designed combustor makes this possible; a less effective combustor can be made to function simply by elevating the static temperature until spontaneous ignition is achieved. Low combustor entry temperature is desirable/essential due to intake and nozzle limitations.

This paper aims in particular at the application of scalar and joint scalar-velocity-turbulent frequency PDF (probability density functions) methods to supersonic combustion done by P. Gerlinger \& H.Mobus et al[4]. Supersonic combustion has the potential of providing propulsion systems for a new generation of air breathing space transportation vehicles. Accuracy is an all-important issue. Supersonic combustion is commonly considered as one of the most demanding applications of current CFD tools.. However, rapid ignitions as well as fast and complete combustion are vital to reduce hardware length and weight. Therefore, hydrogen is the fuel of choice owing to its short ignition delay and, in view of structural mechanics, because of its efficiency in cooling. As a last point it may be concluded that more high-quality experimental data are indispensable for further evaluation of high speed combustion models.

A numerical study of mixing and combustion enhancement has been performed by Peter Gerlinger\&Peter Stoll et al[5] for a Mach 2 . Due to the extremely short 
residence time of the air in supersonic combustors, an efficient (rapid and with small losses in total pressure) fuel/air mixing is hard to achieve. K. Kumaran\&V.Babu [6] investigates the effect of chemistry models on the predictions of supersonic combustion of hydrogen in a model combustor. The calculations show that multi step chemistry predicts higher and wider spread heat release than what is predicted by single step chemistry. In addition, it is also shown that multi step chemistry predicts intricate details of the combustion process such as the ignition distance and induction distance. a detailed chemistry model with 37 reactions and 9 species was used and the results from these calculations were compared with those obtained using single step chemistry.

However, the prediction of the myriad details of the heat release/ignition delay, which offer insights into the combustion process, demands a comprehensive chemistry model as demonstrated in this work.

A numerical study of atomization, i.e. breakup of a high speed jet and spray formation, is presented by Zhiliang Xuxk \& Wohno Ohzk et al [7] using the Front Tracking method in 2D. The high speed flow in the nozzle gives rise to cavitation, i.e. a mixed liquid-vapor region.

A Lagrangian model of turbulent combustion in high speed flows has been used in conjunction with an efficient RANS-AMA strategy to simulate both non-reactive and reactive turbulent supersonic coflowing jets. Liquid hydrocarbon supersonic combustion has been experimentally investigated by C. GRUENIG \& F. Mayinger [9] . Kerosene was burnt in a steady, vitiated Mach 2.15 - air flow of a model scramjet combustor. The fuel is injected into the supersonic air stream by means of pylons. By the addition of small amounts of hydrogen to the kerosene the liqid fuel jet is dispersed and a fine spray produced. However, this additional fuel jet dispersion is not necessary for the supersonic combustion if the fuel is injected normally into the cross flow. combustor ignition behaviour, the air stream temperature can be reduced below the combustor ignition level Tmin once the combustor has ignited. Below Tflame-out the time scale ratio tignition/tresidence reaches its unstable regime again and the flame extinguishes.

Kyung Moo Kim \& Seung Wook Baek et al [10] describes the numerical investigations concerning the combustion enhancement when a cavity is used for the hydrogen fuel injection through a transverse slot nozzle into a supersonic hot air stream. The combustor with cavity is found to enhance mixing and combustion while increasing the pressure loss, compared with the case without cavity. But it is noted that there exists an appropriate length of cavity regarding the combustion efficiency and total pressure loss. Usually, the cavity was found to increase both the total pressure loss and the temperature of the combustor while enhancing the combustion of fuel and oxidizer. A large-eddy simulation (LES) model with a new localized dynamic sub grid closure for the magneto-hydrodynamics (MHD) equations is used to investigate plasma-assisted combustion in supersonic flow by Kenji Miki\&Joey Schulz et al [11]. A 16- species and 74-reactions kinetics model is used to simulate hydrogen-air combustion and high-temperature air dissociation. It is observed that an electrical discharge creates a high temperature and a radical rich concentration region in the recirculation zone that aids in ignition and flame-holding. When an uniform magnetic field is applied, mixing is significantly enhanced since the shock structure ahead of the fuel jet is weakened and fuel penetration into the air cross flow is increased. P Manna\&D Chakraborty[12] shows the Reacting./low field of $\mathrm{H}$ l-air combustion behind a backward fating step in a ,"constant area combustor is simulated numerically by solving three-dimensional Navier Stokes equations along with $\mathrm{K}$-e turbulence model and fast rate chemistry. Investigation of kerosene combustion in a Mach 2.5 flow was carried out using a model supersonic combustor with cross-section area of $51 \mathrm{~mm} * 70 \mathrm{~mm}$ and different integrated fuel injector/flameholder cavity modules is done by G. Yu\&J.G. Li et al[13]. Experiments with pure liquid atomization and with effervescent atomization were characterized and compared. Under the same operation conditions, comparison of the measured static pressure distributions along the combustor also shows that effervescent atomization generally leads to better combustion performance than the use of pure liquid atomization.

Experiments were performed by Chadwick C.\&C. Rasmussena et al [14] to examine the stability of hydrocarbon-fueled flames in cavity flame holders in supersonic airflows. Ethylene flames were stable over a wider range of fuel flowrates than methane flames, as expected because of the shorter ignition delay time and greater flame speed of ethylene. Methane flames would not ignite in a Mach 3 airflow due to the low static temperature and pressure. Lean limits for ethylene in the Mach 2 and Mach 3 cases were comparable, although flames were stabilized over a smaller range of $\mathrm{m} \_\mathrm{A}$ in the Mach 3 airflow. A radially lobed nozzle (petal nozzle) is being increasingly recognised as a potential candidate for promoting mixing in compressible flows. An experimental investigation has been conducted by A. R. SRIKRISHNAN\&J. KURIAN et al [15] to study its effectiveness in improving thermal mixing and combustion in supersonic flow. A hot gas jet issuing supersonically from a lobed nozzle mixes with a cold supersonic jet in a circular mixing tube. The two jets issue coaxially. A detailed survey of the flow field inside the mixing duct reveals that nearly complete thermal mixing (as exemplified by the nearly uniform temperature distribution) could be achieved in a short distance when a lobed nozzle is employed. The results also indicate the presence of large-scale vortices in the flow field downstream of the lobed nozzle. Having thus created a field in which mixing is good, supersonic combustion was then attempted. Kerosene was introduced into the hot stream issuing from the lobed nozzle and it burned mainly in the mixing tube, which served as a supersonic combustor. Resulting temperature and pressure rises were measured and the supersonic combustion efficiency was found to be of the order of $60 \%$. YUAN Shengxue[16] discuss the difference between supersonic combustion and subsonic combustion is discussed, and the mechanism of supersonic combustion propagation and the limitation of heat addition in supersonic flow are pointed out. The results of the calculation of deflagration in supersonic flow show that the entropy increment and the total pressure loss of the combustion products may decrease with the increase of combustion velocity. It is also demonstrated that 
the oblique detonation wave angle may not be controlled by the wedge angle under weak underdriven solution conditions and be determined only by combustion velocity. Therefore, the weak underdriven solution may become self-sustaining oblique detonation waves with a constant wave angle. The effects of dimethyl ether (DME) addition on the high temperature ignition and burning properties of methane-air mixtures were studied experimentally and numerically by Zheng Chen\&Xiao Qin et al[17]. The results showed that for a homogeneous system, a small amount of DME addition to methane resulted in a significant reduction in the high temperature ignition delay. The ignition enhancement effect by DME addition was found to exceed that possible with equivalent amounts of hydrogen addition, and it was investigated by using radical pool growth and computational singular perturbation analysis.

Y. d'Angelo [18] deals with the analysis of the flow structure when a supersonic air-hydrogen mixture encounters a deflection ramp. It should be noted that, in this particular case, a "very hot" region is observed near the point where the 3 shocks meet, region where the temperature is significantly higher than in the portion behind the normal shock. Elementary reactions of interest in $\mathrm{H} 2$ supersonic combustion chemistry have been investigated by S. Javoy\&V Naudet et al[19] using a shock tube technique connected to an atomic resonance absorption spectrophotometer. $\mathrm{He}$ concluded that Resonance absorption measurements of $\mathrm{O}$ atoms, behind reflected shock waves, have been used to precise the rate coefficients of elementary reactions

Which play major role in $\mathrm{H} 2$ supersonic combustion chemistry. H. NAGATA\&M. Sasaki et al [20] propose a new simple method to evaluate hydrogen concentrations in a hydrogen/air supersonic mixing layer without the need for costly apparatus. Catalytic reaction occurs on an electrically heated platinum wire in the supersonic flow of a hydrogen/air mixture. It is found that the effect of hydrogen concentration on the ratio of heat and mass transfer coefficients is very weak, suggesting that the mass transfer coefficient is obtained with reasonable accuracy from the heat transfer coefficient by assuming the equivalent spatial distributions of heat and mass transfer. Based on this result, a method to translate the catalytic heat release rate into the hydrogen concentration of the flow is proposed. To prove the accuracy of this method, hydrogen concentrations of hydrogen/air premixed supersonic flows were measured successfully.

An experimental effort to characterize the flame-holding process of a hydrogen jet injected into a high total enthalpy supersonic cross flow. An expansion tube is used to provide a correct simulation of true flight combustion chemistry, including ignition delay and reaction times. This approach permitted a number of unique experiments involving acceleration of radical-free air to high total enthalpies. The experiments were designed by A. BEN-YAKAR\&R. K. Hanson[21] to map the near-field flow characteristics and auto-ignition process of an under expanded transverse hydrogen jet injected into flight-Mach number 10 and 13 total enthalpy flow conditions.

Analytical and numerical assessments of the indirect noise generated through a nozzle are presented by M. Leyko \& F. Nicoud et al [22]. The configuration corresponds to an experimental setup operated at DLR by Bake et al. (2008) where an entropy wave is generated upstream of the nozzle by means of an electrical heating device. Both 3-D and 2-D axisymmetric simulations are performed to demonstrate that the experiment is mostly driven by linear acoustic phenomena, including pressure wave reflection at the outlet and entropy-to-acoustic conversion in the accelerated regions. Results show that the acoustic impedance downstream of the nozzle must be accounted for appropriately in order to recover the experimental pressure signal. A good agreement is also obtained with a purely analytical assessment based on the Marble and Candel compact nozzle approximation. The entropy-to-acoustic conversion due the strong mean velocity gradient in the nozzle, including the normal shock that stands just downstream of the throat. The acoustic reflection within the exhaust system downstream of the nozzle and test section. Moreover, in the low frequency range investigated, only 1-D planar waves are present and the compact nozzle approximation is valid, even for the entropy perturbations. As a result, the pressure signals observed experimentally and numerically can be nicely reproduced by a simple quasi-1-D analytical model derived in the zero frequency limit for the nozzle.

A numerical analysis was carried out by Doyoung Byun \& Seung Wook Baek[23] in order to investigate the combustion and heat transfer characteristics in a liquid rocket engine in terms of non-gray thermal radiation and soot formation. A counterflow diffusion flame of hydrogen, developed in the forward stagnation-flow region of a porous cylinder in supersonic airflow (which can be considered as a flame of the "Tsuji-burner", in supersonic airflow), was analyzed numerically by Kenichi Takita [24] to solve the two-dimensional Navier-Stokes equations for multispecies. Flame temperature approaches the stagnation temperature in the region of high static temperature and has maximum heat release at a certain static temperature. This is due to the increase of flow velocity and stretch rate based on the increase of static temperature, but appreciable flame extinction does not occur even if the stretch rate becomes much higher, because the stagnation temperature exceeds the critical temperature, which has previously been discussed for a high-temperature subsonic airflow system Numerical investigations have been carried out by J.X. Wen \& B.P. Xu et al[25] for pressurized hydrogen releases via a length of tube into ambient air. The main findings can be summarized as follows:

The air behind the leading shock is shock-heated and mixes with hydrogen in the contact region to form a significant amount of flammable mixture due to the enhanced turbulent mixing. Ignition is firstly initiated inside the tube. With the development of turbulent mixing a partially premixed flame evolves. Significant amount of shock-heated air and well developed partially premixed flames are two major factors providing potential energy to overcome the strong under-expansion and further flow divergence following spouting from the tube. The predictions show that the initial flames can survive at two locations: (1) at the front of the under expanded jet and (2) within a recirculation zone near the tube exit. The latter is most likely to transit to a jet fire. A survey of physical locations of the optimal discharge placement and their common mixture fraction was performed by Wookyung Kim \& Hyungrok Do et al[26] for a JICF. As a 
plasma discharge, highly nonequilibrium ultra-short-pulsed repetitive discharge (USRD) was used, while a methane jet in cross flow was the flame configuration in the current study. As a criterion to determine the optimal discharge placement, the flame duty cycle, defined by the fraction of the total time that the flame is ignited, was used. They found that the flow characteristics, such as adequate jet concentration, low concentration gradient, low scalar fluctuation, and low strain rate, are the primary factors that determine the possibility of flame ignition.

B.E. Milton\& K. Pianthong[28] has summarised work on pulsed, supersonic liquid fuel jets. These have been studied experimentally for low and high range supersonic Mach numbers and simulated for the low Mach numbers only. The numerical studies have used a one-dimensional analysis and the Autodyne code for assessment of the internal pressure rise due to the impact of the driving projectile. For the external jet, the FLUENT code has been used. This allows such things as the mixing layer to be evaluated which is important for further incorporation into engine combustion codes. It must be emphasized that, at this stage, results are still preliminary and more development is required. Supersonic fuel jets may have applications in diesel engine and scramjet technology. Their properties require a fundamental examination as their mixing characteristics are likely to be affected by the leading edge shock wave. Such jets have been created experimentally in the laboratory for $\mathrm{M}=1.8$ to $\mathrm{M}=5.6$ by $\mathrm{S}$. Zakrzewski \& B.E. Milton et al [29] but require further CFD studies to examine details that are obscure in the experiments. The CFD development has examined the shock structure ahead of the jet, and the mixing layer for jets emerging from the nozzle as either vapour or liquid. So far, the jet fluid considered has been water or water vapour. A possibility of a considerable enhancement of ignition in a diffusion mode in a supersonic non-premixed H2-air flow by means of the laser-induced excitation of $\mathrm{O} 2$ molecules to the b1 $\sum \mathrm{g}+$ electronic state is investigated by A.M. Starik \& N.S. Titova et al[30] on the base of numerical simulation. It is shown that for significant shortening the induction zone length it is sufficient to expose only a thin air layer (with thickness of $1-2 \mathrm{~cm}$ ) to resonance laser radiation with a small input radiation energy $5 \times 103-2 \times 102 \mathrm{~J} / \mathrm{cm} 3$. Even a small amount of radiation energy transmitted to the air flow makes it possible to arrange a stable combustion at a short distance $(61 \mathrm{~m})$ from the irradiation region at a relatively low temperature of the air $(\mathrm{T}=800 \mathrm{~K})$. Shortening the induction zone length, in this case, is due to intensification of chain-branching via excitation of $\mathrm{O} 2$ molecules by laser radiation and penetration of excited molecules into the shear layer.

Floor injectors provide advantages near RBO because unburned fuel can escape through the shear layer and it does not readily build up in the cavity. It was demonstrated by Chadwick C.Rasmussen \& Sulabh K. Dhanuka et al [31] that large $\mathrm{CH} 2 \mathrm{O}$ signals can be obtained by increasing the camera gate duration to take advantage of the long fluorescence lifetime of formaldehyde at low pressure.

\section{FORMULATION AND SOLUTION METHODOLOGY}

A mathematical model comprises equations relating the dependent and the independent variables and the relevant parameters that describe some physical phenomenon. Typically, a mathematical model consists of differential equations that govern the behavior of the physical system, and the associated boundary conditions.

\section{GOVERNING EQUATIONS}

The advantage of employing the complete Navier-Stokes equations extends not only o the investigations that can be carried out on a wide range of flight conditions and geometries, but also in the process the location of shock wave, as well as the physical characteristics of the shock layer, can be precisely determined. We begin by describing the three-dimensional forms of the Navier-Stokes equations below. Note that the two-dimensional forms are just simplification of the governing equations in the three dimensions by the omission of the component variables in one of the co-ordinate directions. Neglecting the presence of body forces and volumetric heating, the three-dimensional Navier-Stokes equations are derived as[33]

$$
\begin{aligned}
& \text { Continuity: } \frac{\partial \rho}{\partial t}+\frac{\partial(\rho u)}{\partial x}+\frac{\partial(\rho v)}{\partial y}+\frac{\partial(\rho w)}{\partial z}=0 \\
& \text { X-momentum: } \frac{\partial(\rho u)}{\partial t}+\frac{\partial(\rho u u)}{\partial x}+\frac{\partial(\rho v u)}{\partial y}+\frac{\partial(\rho w u)}{\partial z}= \\
& \frac{\partial \sigma_{x x}}{\partial x}+\frac{\partial \tau_{y x}}{\partial y}+\frac{\partial \tau_{z x}}{\partial z} \\
& \text { y-momentum: } \frac{\partial(\rho v)}{\partial t}+\frac{\partial(\rho u v)}{\partial x}+\frac{\partial(\rho v v)}{\partial y}+\frac{\partial(\rho w v)}{\partial z}= \\
& \frac{\partial \tau_{x y}}{\partial x}+\frac{\partial \sigma_{y y}}{\partial y}+\frac{\partial \tau_{z y}}{\partial z} \\
& \text { z-momentum: } \frac{\partial(\rho w)}{\partial t}+\frac{\partial(\rho w w)}{\partial x}+\frac{\partial(\rho v w)}{\partial y}+\frac{\partial(\rho w w)}{\partial z} \\
& =\frac{\partial \tau_{x z}}{\partial x}+\frac{\partial \tau_{y z}}{\partial y}+\frac{\partial \sigma_{z z}}{\partial z} \\
& \text { energy: } \frac{\partial(\rho E)}{\partial t}+\frac{\partial(\rho u E)}{\partial x}+\frac{\partial(\rho \nu E)}{\partial y}+\frac{\partial(\rho w E)}{\partial z}= \\
& \frac{\partial\left(u \sigma_{x x}+v \tau_{x y}+w \tau_{x z}\right)}{\partial x}+\frac{\partial\left(u \tau_{y x}+v \sigma_{y y}+w \tau_{y z}\right)}{\partial y}+ \\
& \frac{\partial\left(u \tau_{z x}+v \tau_{z y}+w \sigma_{z z}\right)}{\partial z} \\
& +\frac{\partial\left(k \frac{\partial T}{\partial x}\right)}{\partial x}+\frac{\partial\left(k \frac{\partial T}{\partial y}\right)}{\partial y}+\frac{\partial\left(k \frac{\partial T}{\partial z}\right)}{\partial z}
\end{aligned}
$$

Assuming a Newtonian fluid, the normal stress $\sigma x x$, бyy, and $\sigma z z$ can be taken as combination of the pressure $p$ and the normal viscous stress components $\tau \mathrm{xx}, \tau \mathrm{yy}$, and $\tau \mathrm{zz}$ while the remaining components are the tangential viscous stress components whereby $\tau \mathrm{xy}=\tau \mathrm{yx}, \tau \mathrm{xz}=\tau \mathrm{zx}$, and $\tau \mathrm{yz}=\tau \mathrm{zy}$. For the energy conservation for supersonic flows, the specific energy $E$ is solved instead of the usual thermal energy $\mathrm{H}$ applied in sub-sonic flow problems. In three dimensions, the specific energy $\mathrm{E}$ is repeated below for convenience:

$$
E=e+\frac{1}{2}\left(u^{2}+v^{2}+w^{2}\right)
$$

It is evident from above that the kinetic energy term 
contributes greatly to the conservation of energy because of the high velocities that can be attained for flows, where Ma>1. Equations (1)-(6) represent the form of governing equations that are adopted for compressible flows.

The solution to the above governing equations nonetheless requires additional equations to close the system. First, the equation of state on the assumption of a perfect gas in employed, that is,

$$
P=\rho R T,
$$

where $\mathrm{R}$ is the gas constant.

Second, assuming that the air is calorically perfect, the following relation holds for the internal energy:

$$
\mathrm{e}=\mathrm{C}_{\mathrm{v}} \mathrm{T} \text {, }
$$

where $\mathrm{Cv}$ is the specific heat of constant volume. Third, if the Prandtl number is assumed constant (approximately 0.71 for calorically perfect air), the thermal conductivity can be evaluated by the following:

$$
\mathrm{k}=\frac{\mu \mathrm{C}_{\mathrm{p}}}{P_{r}}
$$

The Sutherland's law is typically used to evaluate viscosity $\mu$, which is provided by

$$
\mu=\mu_{0}\left(\frac{T}{T_{0}}\right)^{1.5} \frac{T_{0}+120}{T+120}
$$

where $\mu_{0}$ and $T_{0}$ are reference values at standard sea level conditions.

Generalized form of Turbulence Equations is as follows :

$$
\begin{aligned}
& (k) \frac{\partial k}{\partial t}+\frac{\partial(u k)}{\partial x}+\frac{\partial(v k)}{\partial y}+\frac{\partial(w k)}{\partial z}=\frac{\partial\left[\frac{V_{T}}{\sigma_{k}} \frac{\partial k}{\partial x}\right]}{\partial x}+ \\
& \frac{\partial\left[\frac{V_{T}}{\sigma_{k}} \frac{\partial k}{\partial y}\right]}{\partial y}+\frac{\partial\left[\frac{V_{T}}{\sigma_{k}} \frac{\partial k}{\partial z}\right]}{\partial z}+\left(S_{k}=\mathrm{P}-\mathrm{D}\right) \\
& (\epsilon) \frac{\partial \epsilon}{\partial t}+\frac{\partial(u \epsilon)}{\partial x}+\frac{\partial(v \epsilon)}{\partial y}+\frac{\partial(w e)}{\partial z}=\frac{\partial\left[\frac{V_{T}}{\sigma_{k}} \frac{\partial \epsilon}{\partial x}\right]}{\partial x}+ \\
& \frac{\partial\left[\frac{V_{T}}{\sigma_{k}} \frac{\partial \epsilon}{\partial y}\right]}{\partial y}+\frac{\partial\left[\frac{V_{T}}{\sigma_{k}} \frac{\partial \epsilon}{\partial z}\right]}{\partial z}+\left(S_{\epsilon}=\frac{\epsilon}{k}\left(C_{e 1} P-C_{e 2} D\right)\right.
\end{aligned}
$$

where

$$
\begin{aligned}
& P=2 v_{T}\left[\left(\frac{\partial u}{\partial x}\right)^{2}+\left(\frac{\partial v}{\partial y}\right)^{2}+\left(\frac{\partial w}{\partial z}\right)^{2}\right]+v_{T}\left[\left(\frac{\partial u}{\partial y}+\frac{\partial v}{\partial x}\right)^{2}+\left(\frac{\partial v}{\partial z}+\frac{\partial w}{\partial y}\right)^{2}+\right. \\
& \left.\left(\frac{\partial w}{\partial x}+\frac{\partial u}{\partial z}\right)^{2}\right] \text { and } D=\epsilon
\end{aligned}
$$

\section{Combustion Modeling}

The eddy dissipation combustion model is used to simulate The reactions in flames. The eddy dissipation combustion model[32], used .extensively for its simplicity and robustness in predicting reactive flows, is based on the concept that chemical reaction is very fast relative to the transport process in the flow: The products are formed instantaneously as the reactants mix at the molecular level. The model assumes that the reaction rate may be related directly to the time required to mix reactants at molecular level. In turbulent flows, this mixing time is dictated by the eddy properties. And therefore, the burning rate in proportional to the rate at which turbulent Hot Air kinetic energy is dissipated, that is, reaction rate $\alpha$ $\varepsilon / K$, where $K$ is the turbulent kinetic energy and $\varepsilon$ is its dissipation rate. The chemistry of the combustion reaction of hydrogen in air is represented on a molar basis by

$$
\mathrm{H}_{2}+1 / 2 \mathrm{O}_{2}=\mathrm{H}_{2} \mathrm{O} \text {. }
$$

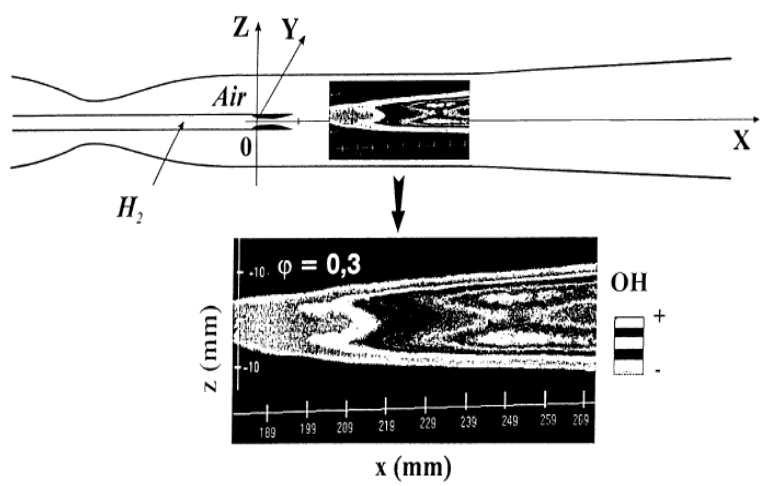

Figure1 supersonic combustion experiment and visualization of the $\mathrm{OH}[32]$

\section{SCOPE OF FURTHER STUDY: -}

The investigation can be performed further in the following areas,

1. To design a contour to make the flow at Mach number 2 for various higher Mach number for supersonic combustion.

2. To analysis the pressure, temperature, velocity as well as turbulence kinetic energy in the combustion chamber.

3. To check the flow properties in combustion chamber and its effect by using the variety of fuel.

4. To check the flow properties in combustion chamber and its effect by using the variety of mixture fuel.

5. Indirect combustion in exhaust gases by using the secondary air-fuel at the exit of the duct.

6. To analysis the flow characteristics at various degree of angle for different no. of fuel injectors.

7. Evaluation of mass fraction for various fuel and there mixture in the combustion chamber.

8. Study on industrial flows, CFD analysis of flow in internal combustion chambers, and heat transfer analysis from fluent and Ansys packages.

9. To see the CFD analysis of the flow from mufflers.

\section{CONCLUSION}

In this field many researchers worked to develop a configuration giving efficient mixing and combustion, also meeting the requirements of flame holding and completion of combustion with sufficient stabilization in the flow field. The main attention is paid to the local intensity of heat release, which determines, together with the duct geometry, techniques for flame initiation and stabilization, injection techniques and quality of mixing the fuel with oxidizer, the gas-dynamic flow regime. From the survey we concluded that some area in which more attention to be paid like total pressure loss in combustion chamber and design a contour to make the flow at Mach no. 2 for various higher Mach no. for supersonic combustion and analyze the flow properties in combustion chamber and its effect by using the variety of 
fuel with the help of CFD tool.

\section{ACKNOWLEDGEMENT}

The authors acknowledge the valuable suggestions from Prof. E. Rathakrishnan, Professor, Department of aerospace engineering, IIT Kanpur, India. The authors acknowledge the financial help provided by AICTE from the project AICTE: 8023/RID/BOIII/NCP(21) 2007-2008 .The Project id at IIT Guwahati is ME/P/USD/4.

\section{REFERENCES}

[1] P.K. Tretyakov "The Problems Of Combustion At Supersonic Flow" West-East High Speed Flow Field Conference 19-22, November 2007.

[2] Shigeru Aso, Arifnur Hakim, Shingo Miyamoto, Kei Inoue And Yasuhiro Tani "Fundamental Study Of Supersonic Combustion In Pure Air Flow With Use Of Shock Tunnel”, Acta Astronautica 57 (2005) $384-389$.

[3] T. Cain And C. Walton "Review Of Experiments On Ignition And Flame Holding In Supersonic Flow" Published By The America Institute Of Aeronautics And Astronautics, Rto-Tr-Avt-007-V2.

[4] H. Mo"Bus, P. Gerlinger, D. Bru"Ggemann "Scalar And Joint ScalarVelocity- Fequency Monte Carlo Pdf Simulation Of Supersonic Combustion" Combustion And Flame 132 (2003) 3-24.

[5] Peter Gerlinger, Peter Stoll 1, Markus Kindler, Fernando Schneider C, Manfred Aigner "Numerical Investigation Of Mixing And Combustion Enhancement In Supersonic Combustors By Strut Induced Streamwise Vorticity", Aerospace Science And Technology 12 (2008) 159-168.

[6] K. Kumaran, V. Babu "Investigation Of The Effect Of Chemistry Models On The Numerical Predictions Of The Supersonic Combustion Of Hydrogen", Combustion And Flame 156 (2009) 826-841.

[7] Zhiliang Xuxk, Myoungnyoun Kimxk, Wonho Ohzk, James Glimmzxk, Roman Samulyakx, Xiaolin Liz And Constantine Tzanos "Atomization Of A High Speed Jet" April 14, 2005, U.S. Department Of Energy

[8] Jean-Francois Izard *, Arnaud Mura "Stabilization Of Non-Premixed Flames In Supersonic Reactive Flows" Combustion For Aerospace Propulsion, Cras2b:2818,

[9] C. Gruenig* And F. Mayinger "Supersonic Combustion Of Kerosene/H2-Mixtures In A Model Scramjet Combustor", Institute A For Thermodynamics, Technical University Munich, D-85747

[10] Kyung Moo Kim 1, Seung Wook Baek *, Cho Young Han "Numerical Study On Supersonic Combustion With Cavity-Based Fuel Injection", International Journal Of Heat And Mass Transfer 47 (2004) 271-286,

[11] Kenji Miki, Joey Schulz, Suresh Menon "Large-Eddy Simulation Of Equilibrium Plasma-Assisted Combustion In Supersonic Flow", Proceedings Of The Combustion Institute 32 (2009) 2413-2420, Atlanta, Ga 30332-0150,

[12] P Manna, D Chakraborty "Numerical Simulation Of Transverse H2 Combustion In Supersonic Airstream In A Constant Area Duct", Vol. 86, November 2005, ARTFC.

[13] G. Yu, J.G. Li, J.R. Zhao, L.J. Yue, X.Y. Chang, C.-J. Sung “An Experimental Study Of Kerosene Combustion In A Supersonic Model Combustor Using Effervescent Atomization", Proceedings Of The Combustion Institute 30 (2005) 2859-2866,

[14] Chadwick C. Rasmussena, James F. Driscolla, Kuang-Yu Hsub, Jeffrey M. Donbarc, Mark R. Gruberc, Campbell D. Carter "Stability Limits Of Cavity-Stabilized Flames In Supersonic Flow", Proceedings Of The Combustion Institute 30 (2005) 2825-2833.

[15] A. R. Srikrishnan , J. Kurian And V. Sriramulu "An Experimental Investigation Of Thermal Mixing And Combustion In Supersonic Flows", Combustion And Flame 107:464-474 (1996).

[16] Yuan Shengxue, "On Supersonic Combustion", Voi. 42 No. 2, Science In China (Series A) February 1999.

[17] Zheng Chen, Xiao Qin, Yiguang Ju *, Zhenwei Zhao, Marcos Chaos, Frederick L. Dryer, "High Temperature Ignition And Combustion Enhancement By Dimethyl Ether Addition To Methane-Air Mixtures", Proceedings Of The Combustion Institute 31 (2007) 1215-1222.

[18] Y. D'angelo, "Computation Of The Flow Structure Of An Hydrogen/Air Mixture Downstream Of A Steady System Of Shock Waves", Aerospace Science And Technology, 1997, No 5, 309-327.
[19] S. Javoy, V. Naudet, S. Abid, C.E. Paillard, "Elementary Reaction Kinetics Studies Of Interest In H2 Supersonic Combustion Chemistry", Experimental Thermal And Fluid Science 27 (2003) 371-377.

[20] H. Nagata, M. Sasaki, T. Arai, T. Totani, And I. Kudo, "Evaluation Of Mass Transfer Coefficient And Hydrogen Concentration In Supersonic Flow By Using Catalytic Reaction", Proceedings Of The Combustion Institute, Volume 28, 2000/Pp. 713-719,

[21] A. Ben-Yakar And R. K. Hanson, "Experimental Investigation Of Flame-Holding Capability Of Hydrogen Transverse Jet In Supersonic Cross-Flow", Twenty-Seventh Symposium (International) On Combustion/The Combustion Institute, 1998/Pp. 2173-2180,

[22] M. Leyko, F. Nicoud, S. Moreau And T. Poinsot, "Numerical And Analytical Investigation Of The Indirect Combustion Noise In A Nozzle", Combustion For Aerospace Propulsion, Cras2b:2838.

[23] Doyoung Byun And Seung Wook Baek, "Numerical Investigation Of Combustion With Non-Gray Thermal Radiation And Soot Formation Effect In A Liquid Rocket Engine", International Journal Of Heat And Mass Transfer 50 (2007) 412-422,

[24] Kenichi Takita, "Numerical Simulation Of A Counterflow Diffusion Flame In Supersonic Airflow", Twenty-Sixth Symposium (International) On Combustion/The Combustion Institute, 1996/Pp. 2877-2883.

[25] J.X. Wen*, B.P. Xu And V.H.Y. Tam, "Numerical Study On Spontaneous Ignition Of Pressurized Hydrogen Release Through A Length Of Tube", Combustion And Flame 2009.

[26] Wookyung Kim, Hyungrok Do, M. Godfrey Mungal And Mark A. Cappelli, "Optimal Discharge Placement In Plasma-Assisted Combustion Of A Methane Jet In Cross Flow", Combustion And Flame 153 (2008) 603-615.

[27] Valeriyi.Timoshenko, Igors.Belotserkovets And Vjacheslavp.Gusinin, "Problems Of Providing Completeness Of The Methane-Containing Block-Jet Combustion In A Rocket-Ramjet Engine's Combustion Chamber", Acta Astronautica .2009.03.033.

[28] B.E. Milton And K. Pianthong, "Pulsed, Supersonic Fuel Jets-A Review Of Their Characteristics And Potential For Fuel Injection", International Journal Of Heat And Fluid Flow 26 (2005) 656-671.

[29] S. Zakrzewski, B.E. Milton, K. Pianthong And M. Behnia, "Supersonic Liquid Fuel Jets Injected Into Quiescent Air", International Journal Of Heat And Fluid Flow 25 (2004) 833-840.

[30] A.M. Starik, N.S. Titova, L.V. Bezgin, And V.I. Kopchenov, "The Promotion Of Ignition In A Supersonic H2-Air Mixing Layer By Laser-Induced Excitation Of O2 Molecules: Numerical Study", Combustion And Flame 156 (2009) 1641-1652.

[31] Chadwick C. Rasmussen, Sulabh K. Dhanuka, And James F. Driscoll, "Visualization Of Flameholding Mechanisms In A Supersonic Combustor Using Plif", Proceedings Of The Combustion Institute 31 (2007) 2505-2512.

[32] P. Magre, G. Collin, O. Pin, J.M Badie, G. Olalde And M. Clement, Temperature Measurement By Cars And Intrusive Probe In An Air-Hydrogen Supersonic Combustion, International Journal Of Heat And Mass Transfer 44(2001) 4095-4105.

[33] Jiyun tu, guan Heng yeoh and chaoqun liu. "Computational Fluid Dynamics" Elsevier Inc. 2008.

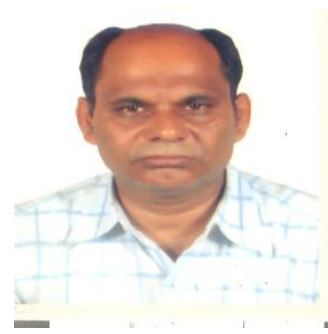

Dr. K.M.Pandey did his PhD in Mechanical Engineering in 1994 from IIT Kanpur. He has published and presented 170 papers in International \& National Conferences and Journals. Currently he is working as Professor of the Mechanical .Engineering Department, National Institute of Technology, Silchar, Assam, India..He also served the department in the capacity of head from July 07 to 13 July 2010. He has also worked as faculty consultant in Colombo

Plan Staff College, Manila, Philippines as seconded faculty from Government of India. His research interest areas are the following; Combustion, High Speed Flows, Technical Education, Fuzzy Logic and Neural Nnetworks, Heat Transfer, Internal Combustion Engines, Human Resource Management, Gas Dynamics and Numerical Simulations in CFD area from Commercial Softwares. Email:kmpandey2001@yahoo.com. 